\title{
Spectum Sharing as Congestion Games
}

\author{
Mingyan Liu*, Yunnan $\mathrm{Wu}^{\dagger}$ \\ *Dept. of EECS, University of Michigan, Ann Ärbor, MI 48105, mingyan@eecs. umich. edu \\ ${ }^{\dagger}$ Microsoft Research, Redmond, WA 98052, yunnanwu@microsoft.com
}

\begin{abstract}
A fundamental problem in wireless networking is efficient spectrum sharing. In this paper we study this problem in the context of decentralized multi-user frequency adaptation, with the objective of designing protocols that are efficient, agile, robust, and incentive-compatible. Our approach is based on the theory of congestion games, a class of games that models the competition for resources among multiple selfish players. In a congestion game, when a player unilaterally switches her strategy, the change in her own payoff is the same as the change in a global objective known as the potential function. Hence any sequence of unilateral improvements results in a pure strategy Nash equilibrium. In other words, the game is such that selfish behaviors collectively result in a socially desirable outcome. Motivated by the attractive properties of congestion games, this paper sets out to understand how this framework can be used to construct efficient spectrum sharing protocols.

The key challenge in casting spectrum sharing as a congestion game lies in the proper definition of resources. Simply treating wireless channels as resources fails to capture the effect of spatial reuse. We first show how to reformulate two existing distributed spectrum sharing protocols as congestion games. Such reformulation is done by introducing virtual resources that model pair-wise interference. We then provide a new formulation by treating frequency-space blocks as resources. We use this formulation to construct practical protocols for spectrum sharing between multiple base stations/access points. Different implementation methods based on different signaling assumptions are discussed. We further demonstrate that the proposed approach can be readily extended in several aspects, including the modeling of channel bundling and fractional frequency reuse.
\end{abstract}

\section{INTRODUCTION}

We consider a wireless communication system with multiple users, each having access to a common set of channels. A user can only access one channel at a time, but can switch between channels. If multiple users access the same channel at the same time, they will experience potentially degraded performance. Our principle interest lies in optimizing the system wide performance and spectrum efficiency, via effective user sharing mechanisms.

This and similar problems have recently captured increasing interest from the research community, particularly in the context of cognitive radio networks (CRN) and software defined ratio (SDR) technologies, whereby devices are expected to have far greater flexibility in sensing channel availability/condition and moving operating frequencies.

Consider the multi-user multi-channel access system outlined above, and if we limit our attention to a fully decentralized scenario where each user observes the system and makes its own decision, without coordination by a central controller, e.g., a spectrum manager, then a natural way of studying such a system is to model it as a noncooperative game. Examples include [6], [7], [10], some of which will be discussed further below.

For the problem considered in this paper, of particular relevance and interest is a class of strategic games known as congestion games (CG) [1], [2]. A congestion game $\Gamma$ is given by the tuple $\left(\mathcal{N}, \mathcal{R},\left(\Sigma_{i}\right)_{i \in \mathcal{N}},\left(g_{r}\right)_{r \in \mathcal{R}}\right)$, where $\mathcal{N}=\{1,2, \cdots, N\}$ denotes a set of players/users, $R=$ $\{1,2, \cdots, R\}$ the set of resources, $\Sigma_{i} \subset 2^{\mathcal{R}}$ the strategy space of player $i$, and $g_{r}: \mathrm{N} \rightarrow \mathrm{Z}$ a payoff (or cost) function associated with resource $r$. Specifically $g_{r}$ is a function of the total number of users of resource $r$. A player in this game aims to maximize (minimize) its total payoff (cost) which is the sum over all resources its strategy involves. More detailed and formal description of this class of games are provided in Section II.

The congestion game framework is well suited to model resource competition where the resulting payoff (cost) is a function of the level of congestion (number of active users). Congestions games are closely related to potential games [4], and enjoy some remarkable features. In particular, a congestion game is an exact potential game as it admits an exact potential function [2]. Finding a solution (Nash equilibrium or $\mathrm{NE}$ ) to a congestion game is equivalent to finding a (local) optimal solution to this potential function. It is also known that any improvement path is finite (in which each player's improvement move also improves the potential) and leads to a pure strategy NE. In other words, even though the system is decentralized and all players are selfish, by seeking to optimize their individual objective they end up optimizing a global objective, the potential function, and do so in a finite number of steps regardless of the updating sequence. Therefore if the potential function of a particular congestion game has a meaningul and desirable physical interpretation, then the solution (an NE) to this decentralized game has certain built-in performance guarantee ${ }^{1}$, as it is also a local optimal solution to a global objective.

Congestion games have been extensively studied within the context of network routing, see for instance the network congestion game studied in [5], where source nodes seek minimum delay path to a destination and the delay of a link depends on the number of flows going through that link. However, the standard congestion game fails to capture two critical aspects of resource sharing in wireless communication: interference and spatial reuse. A key assumption underlying the congestion game model is that all users have

\footnotetext{
${ }^{1}$ It is known that in general an NE can be fairly inefficient with respect to a given global objective function.
} 
an equal impact on the congestion, and therefore all that matters is the total number of users of a resource ${ }^{2}$. This however is not true in wireless communication. Specifically, if we consider bandwidth or channels as resources, then sharing the same channel is complicated by pair-wise interference; a user's payoff (e.g., channel quality, achievable rates, etc.) depends on who the other users are and how much interference it receives from them. If all other simultaneous users are located sufficiently far away, then sharing may not cause any performance degradation, a feature commonly known as spatial reuse.

The above consideration poses significant challenge in using the congestion game model, and in some cases may render the latter inapplicable. On the other hand, congestion games possess attractive properties as well as an appealing, physical interpretation that we would like to exploit. For these reasons, we set out to see to what extent this framework may be used in our multi-channel access context. Specifically, we will examine what types of user objectives would allow us to formulate the problem as congestion games, while taking into account the impact of pair-wise interference and spatial reuse.

The key to our methodology is a novel concept referred to below as resource expansion, where we introduce virtual resources that capture the spectral-spatial feature of resources in wireless communication, which in turn allow us to capture interference. In what follows we will first demonstrate the utility of this method by taking from existing literature two problem scenarios and "reverse-engineering" them to equivalent congestion games, thereby showing that (1) stability and optimality results can be obtained automatically following this mapping, and (2) these problems can be made a lot more general by drawing from known results on congestion games. The details on this are given in Section III. We then use the concept of resource expansion to study ("forwardengineer") a spectrum allocation problem for base stations. This is presented in Section IV.

It has to be mentioned that the role of interference in a wireless system has been studied within the context of other classes of games, most notably the well-known Gaussian interference game [6], [12]. In a Gaussian interference game, a player can spread a fixed amount of power arbitrarily across a continuous bandwidth, and tries to maximize its total rate in a Gaussian interference channel over all possible power allocation strategies. It has been shown [6] that it has a pure strategy NE, but it can be quite inefficient; playing a repeated game can improve the performance. In addition [7] investigated a market based power control mechanism via supermodularity, while [10] studied the Bayesian form of the Gaussian interference game in the case of incomplete information.

By contrast, in our problem the total power of a user is not divisible, and it can only use it in one channel at a time. This set up is more appropriate for scenarios where the channels

\footnotetext{
${ }^{2}$ This function may be user-specific (see for example the one studied in [3]), but it remains a function of the total number of active users of that resource.
}

have been pre-defined, and the users do not have the ability to access multiple channels simultaneously (which is the case with many existing devices). Thus in our problem the set of admissible user strategies is a subset of that of the Gaussian interference game. As we will see this difference results in special properties that allow us to obtain stronger results and more insight compared to the latter.

The organization of the remainder of this paper is as follows. In Section II we present a brief review of the literature on congestion games, and motivate the idea behind resource expansion. We then show how some problems can be converted into equivalent congestion games through resource expansion in Section III, and present a specific spectrum sharing problem in Section IV. We discuss extensions of our approach and conclude the paper in Sections V and VI, respectively.

\section{Congestion Games: A Review and Motivation FOR RESOURCE EXPANSION}

In this section we provide a brief review on the definition of congestion games, their relation to potential games and their known properties ${ }^{3}$. We then discuss why the standard congestion game does not take into account interference and spatial reuse, and motivate our resource expansion methodology.

\section{A. Congestion games, potential games: a review}

Congestion games [1], [2] are a class of strategic games given by the tuple $\left(\mathcal{N}, \mathcal{R},\left(\Sigma_{i}\right)_{i \in \mathcal{N}},\left(g_{r}\right)_{r \in \mathcal{R}}\right)$, where $\mathcal{N}=$ $\{1,2, \cdots, N\}$ denotes a set of users, $R=\{1,2, \cdots, R\}$ a set of resources, $\Sigma_{i} \subset 2^{\mathcal{R}}$ the strategy space of player $i$, and $g_{r}: \mathbf{N} \rightarrow \mathbf{Z}$ a payoff (or cost) function associated with resource $r$. The payoff (cost) $g_{r}$ is a function of the total number of users using resource $r$ and in general assumed to be non-increasing (non-decreasing). A player in this game aims to maximize (minimize) its total payoff (cost) which is the sum total of payoff (cost) over all resources its strategy involves.

If we denote by $\sigma=\left(\sigma_{1}, \sigma_{2}, \cdots, \sigma_{N}\right)$ the strategy profile, where $\sigma_{i} \in \Sigma_{i}$, then user $i$ 's total payoff (cost) is given by

$$
g^{i}(\sigma)=\sum_{r \in \sigma_{i}} g_{r}\left(n_{r}(\sigma)\right)
$$

where $n_{r}(\sigma)$ is the total number of users using resource $r$ under the strategy profile $\sigma$.

Rosenthal's potential function $\phi: \Sigma_{1} \times \Sigma_{2} \times \cdots \times \Sigma_{n} \rightarrow \mathbf{Z}$ is defined as

$$
\begin{aligned}
\phi(\sigma) & =\sum_{r \in \mathcal{R}} \sum_{i=1}^{n_{r}(\sigma)} g_{r}(i) \\
& =\sum_{i=1}^{N} \sum_{r \in \sigma_{i}} g_{r}\left(n_{r}^{i}(\sigma)\right),
\end{aligned}
$$

\footnotetext{
${ }^{3}$ This review along with some of our notations are primarily based on references [1], [2], [4].
} 
where the second equality comes from exchanging the two sums and $n_{r}^{i}(\sigma)$ denotes the number of players using resource $r$ whose indices do not exceed $i$ (i.e., in the set $\{1,2, \cdots, i\}$ ).

Now consider player $i$, who unilaterally moves from strategy $\sigma_{i}$ (corresponding to the profile $\sigma$ ) to strategy $\sigma_{i}^{\prime}$ (corresponding to the profile $\sigma^{\prime}$ ). The potential changes by

$$
\begin{aligned}
& \sum_{r \in \sigma_{i}^{\prime}, r \notin \sigma_{i}}^{\Delta \phi\left(\sigma_{i} \rightarrow \sigma_{i}^{\prime}\right)} g_{r}\left(n_{r}(\sigma)+1\right)-\sum_{r \in \sigma_{i}, r \notin \sigma_{i}^{i}} g_{r}\left(n_{r}(\sigma)\right) \\
= & \sum_{r \in \sigma_{i}^{\prime}} g_{r}\left(n_{r}\left(\sigma^{\prime}\right)\right)-\sum_{r \in \sigma_{i}} g_{r}\left(n_{r}(\sigma)\right) \\
= & g^{i}\left(\sigma^{-i}, \sigma_{i}^{\prime}\right)-g^{i}\left(\sigma^{-i}, \sigma_{i}\right),
\end{aligned}
$$

where the second equality comes from the fact that for resources that are used by both strategies $\sigma_{i}$ and $\sigma_{i}^{\prime}$ there is no change in their total number of users. The above result may be obtained either directly from Rosenthal's potential definition (2), or more easily, from the change of sums equation (3) by assuming we are considering the $N$-th player.

The above result shows that the gain (loss) caused by any player's unilateral move is exactly the same as the gain (loss) in the potential, which may be viewed as a global objective function. Since the potential of any strategy profile is finite, it follows that every sequence of improvement steps is finite, known as the finite improvement property (FIP), and they converge to a pure strategy Nash Equilibrium. This NE is a local maximum (minimum) point of the potential function $\phi$, defined as a strategy profile where changing one coordinate cannot result in a greater value of $\phi$.

To summarize, we see that in this game, any sequence of unilateral improvement steps converges to a pure strategy $\mathrm{NE}$, which is also a local optimum point of a global objective given by the potential function.

The $\phi()$ defined above is called an exact potential function, where individual payoff (cost) change as a result of a unilateral move is exactly reflected in this global function:

$$
g^{i}\left(\sigma^{-i}, \sigma_{i}^{\prime}\right)-g^{i}\left(\sigma^{-i}, \sigma_{i}\right)=\phi\left(\sigma^{-i}, \sigma_{i}^{\prime}\right)-\phi\left(\sigma^{-i}, \sigma_{i}\right) .
$$

More generally, a function $P$ is called an ordinal potential function if we have $g^{i}\left(\sigma^{-i}, \sigma_{i}^{\prime}\right) \geq g^{i}\left(\sigma^{-i}, \sigma_{i}\right) \Leftrightarrow$ $\phi\left(\sigma^{-i}, \sigma_{i}^{\prime}\right) \geq \phi\left(\sigma^{-i}, \sigma_{i}\right)$. Games that possess the above properties are called exact potential games and ordinal potential games, respectively.

A congestion game is thus an exact potential game. In [4] it was shown that every potential game may be converted into an equivalent congestion game. However, this conversion process, while powerful in its generality, is rather cumbersome to follow and insights are easily lost. For this reason, we will primarily follow the congestion game framework in our development in the rest of this paper.

\section{B. Resource Expansion}

Our objective is to construct efficient distributed spectrum sharing schemes based on the theory of congestion games. The key here lies in the proper definition of resources. In a multi-user, multi-channel access problem, it is natural to

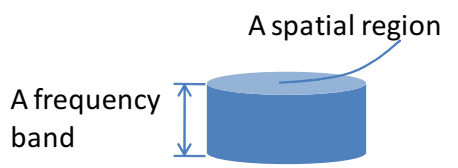

Fig. 1. Illustration of a frequency-space block. The $(x, y)$ dimensions describe a region in space (for visualization purpose we consider space as two-dimensional) and the $z$-dimension describes a frequency band.

think of channels/spectrum bands as resources. The unique feature of spectrum sharing is such that the gain (or cost) of a particular user is a function of the set of other simultaneous users of the same spectrum, rather than the total number of users, as is the basic assumption underlying a congestion game. Players' locality affects the individually perceived interference; far-way users may share the same spectrum without any loss in performance. Therefore if we simply take spectrum as the resource the standard congestion game framework does not directly apply to our problems.

This motivates us to seek alternative definitions of resources. In essence, the true resource in a wireless system is certain spectrum-space-time unit, due to the spatial reuse feature. This is echoed in the way spectrum is traded as commodities. For example, in FCC's spectrum auctions, each spectrum license is the right to use a spectrum band for a certain geographic region (e.g., a city) for a certain period of time (e.g., 10 years). Since we aim at designing decentralized protocols where the users adapt their frequency bands over time, we will drop the time dimension and treat a certain spectrum-space unit as the resource. In short, to cast spectrum adaptation as congestion games, we shall use a set of resources that capture both spectrum and space, expanding the set of physical channels.

It is important to note that there are multiple ways to take space into account when defining the resources. Different ways of accounting for space will imply different coordination methods and lead to different spectrum sharing protocols. As a simple example, we can model the resource consumed by a transmitter-receiver pair as a spectrum-space block illustrated in Figure 1, whose spectrum dimension specifies the spectrum band it is using and the space dimensions specify the interference range of this pair. In what follows we will see three definitions of resources and how they lead to different implementations.

For the rest of this paper, the term player or user specifically refers to a pair of transmitter and receiver in the network. Interference in this context is between one user's transmitter and another user's receiver. This is commonly done in the literature, see for instance [6]. We will also assume that each player has a fixed transmit power.

\section{Resource Expansion: Two ExAmples}

In this section we draw from two existing studies, [11] and [9], respectively, both aimed at designing distributed and stable mechanisms that minimize interference, and show how using resource expansion we can transform them into equivalent congestion games, and thereby making immediately 
available results such as convergence and local optimality.

\section{A. Example One}

In [11] the following multi-user, multi-channel access problem was studied. The users' primary interest is to select a channel such that its total received interference is minimized. Specifically, users $i$ and $j$ each perceive interference $I(i, j)=\frac{P_{o}}{\left(d_{i, j}\right)^{\alpha}}$, where $d_{i, j}$ is the distance between the two and $\alpha$ the passloss exponent, if they happen to select the same channel, and 0 otherwise. A user's objective is to select a channel such that its own total received interference (over all other users in the system) is minimized. It was shown in [11] that greedy user update leads to a local optimal solution to the system objective of total interference.

Below we show how this is indeed a congestion game. Since interference in this case is pair-wise dependent, we will consider a link, defined by a pair of users, combined with a channel, as a (virtual) resource. Specifically, we will define a resource as the triple $(i, j, c)$ where $i, j$ denote an ordered pair of nodes, or equivalently a directed link, and $c$ denotes a channel. Any time when a user $i$ selects channel $c$, it counts as one user of all the resource triples that contain $i$ as one of the two users. With this generalized notion of resource, or resource expansion, the components of the game are listed as follows.

- A set of users/player $\mathcal{N}=\{1,2, \cdots, N\}$.

- A set of channels $\mathcal{C}=\{1,2, \cdots, C\}$.

- A set of resources $\mathcal{R}=\{(i, j, c): i, j \in \mathcal{N}, i \neq j, c \in$ $\mathcal{C}\}$, where $(i, j)$ is an ordered pair of users.

- Pair-wise interference relationship: each pair of user $i, j$ is associated with an interference parameter (a real number) $d_{i, j}$, and $d_{i, j}=d_{j, i}$. This may describe the distance/signal attenuation between the pair of users, or in the special case of $d_{i, j} \in\{0,1\}$, it may describe a binary interference relationship where $d_{i, j}=0$ means the two users do not interfere with each other, and they do otherwise.

- Strategy space $\left(\Sigma_{i}\right)_{i \in \mathcal{N}}$ : a player $i$ has $C$ admissible strategies, each given by the set of resources it consumes:

$$
\begin{array}{r}
\sigma_{i} \in \Sigma_{i}=\{\{(i, j, c),(j, i, c): \forall j \in \mathcal{N}, j \neq i\}, \\
c=1,2, \cdots, C\} .
\end{array}
$$

For convenience, we will use $c\left(\sigma_{i}\right)$ to denote the channel that $i$ uses under strategy $\sigma_{i}$, i.e., this is the value of the last element of the resource triple. We have assumed here that the user is only allowed one channel at a time, but this assumption can be relaxed.

- Using resource $(i, j, c)$ incurs a payoff (cost) of $g_{(i, j, c)}\left(n_{(i, j, c)}\right)$, where $n_{(i, j, c)}$ is the total number of users using resource $(i, j, c)$.

- Under the strategy profile $\sigma=\left(\sigma_{1}, \sigma_{2}, \cdots, \sigma_{N}\right)$ user $i$ gets a total payoff (cost) of

$$
\begin{aligned}
g^{i}(\sigma)= & \sum_{\substack{j \in \mathcal{N}, j \neq i\\
}}\left(g_{\left(i, j, c\left(\sigma_{i}\right)\right)}\left(n_{\left(i, j, c\left(\sigma_{i}\right)\right)}(\sigma)\right)\right. \\
& +g_{\left(j, i, c\left(\sigma_{i}\right)\right)}\left(n_{\left(j, i, c\left(\sigma_{i}\right)\right)}(\sigma)\right)
\end{aligned}
$$

where $n_{(i, j, c)}(\sigma)$ is the total number of players using resource $(i, j, c)$ under the strategy profile $\sigma$.

- Each user's objective is to maximize (minimize) his individual payoff (cost).

The above description obviously defines a congestion game (compare to the description in Section II). We now show that this is the same problem studied in [11].

We define the cost function for each virtual resource as follows, noting that a resource $(i, j, c)$ has at most 2 simultaneous users since each user can only play one strategy at a time.

$$
g_{(i, j, c)}(k)= \begin{cases}\frac{1}{2} f\left(d_{i, j}\right)=\frac{1}{2} \frac{P_{o}}{\left(d_{i, j}\right)^{\alpha}} & k=2 \\ 0 & \text { otherwise }\end{cases}
$$

Obviously we have $g_{(i, j, c)}=g_{(j, i, c)}$. Substituting the above in (7) gives the total cost for user $i$ under the strategy profile $\sigma:$

$$
\begin{aligned}
g^{i}(\sigma) & =\sum_{j: j \neq i, c\left(\sigma_{j}\right)=c\left(\sigma_{i}\right)}\left(g_{\left(i, j, c\left(\sigma_{i}\right)\right)}(2)+g_{\left(j, i, c\left(\sigma_{i}\right)\right)}(2)\right) \\
& =\sum_{j: j \neq i, c\left(\sigma_{j}\right)=c\left(\sigma_{i}\right)} \frac{P_{o}}{\left(d_{i, j}\right)^{\alpha}}
\end{aligned}
$$

which is user $i$ 's sum interference. Therefore under this congestion game, the individual users try to minimize their own perceived sum interference, which is exactly the greedy update proposed in [11].

By Rosenthal's definition, the potential function of this congestion game is given by

$$
\begin{aligned}
\phi(\sigma) & =\sum_{(i, j, c) \in \mathcal{R}} \sum_{k=1}^{n_{(i, j, c)}(\sigma)} g_{(i, j, c)}(k) \\
& =\frac{1}{2} \sum_{i=1}^{N} \sum_{j \neq i: c\left(\sigma_{i}\right)=c\left(\sigma_{j}\right)}\left(g_{\left(i, j, c\left(\sigma_{i}\right)\right)}(2)+g_{\left(j, i, c\left(\sigma_{i}\right)\right)}(2)\right) \\
& =\frac{1}{2} \sum_{i=1}^{N} \sum_{j \neq i} \frac{P_{o}}{\left(d_{i, j}\right)^{\alpha}} I(j \text { picks the same channel as } i)
\end{aligned}
$$

which is a constant factor $(1 / 2)$ of the total amount of interference in the system . This is obviously equivalent to the global objective of total interference used in [11].

We therefore have demonstrated that the congestion game posed above is equivalent to the sum-interference minimization problem studied in [11]. Invoking existing results on congestion games, we immediately have that (1) the above potential function is reduced each time a unique player deviates its strategy to reduce its own received interference, and any updating sequence is finite and converges to an NE of the game; and (2) greedy user updates in any sequence stabilize the system and the stable point is a local minimum of the global objective function: the total interference in the system.

In addition, using congestion game theory we can easily relax two key assumptions adopted in [11]. The first is that interference is symmetric (i.e., $d_{i, j}=d_{j, i}$ ). This assumption may be relaxed by adopting user-specific payoff 
(cost) functions. As was shown in [3], congestion games with user specific payoff functions in general do not have an exact potential function or the FIP property, but nevertheless always possess a pure strategy NE when we limit the number of resources each strategy can involve to one. A second assumption that may be relaxed is that users have access to all channels but can use only one at a time. This assumption can be immediately removed (i.e., each user may have access to a different set of channels and can use multiple channels, or do channel bundling, at a time) within the congestion game framework, without losing any of the above features and conclusions (slightly weaker conclusion if the interference is also assumed to be asymmetric as indicated above).

To summarize, we have reverse-engineered the above problem as a congestion game and reproduced identical results to that in [11]. The advantage and power of casting this problem as a congestion game is that (1) the results follow naturally and straightforwardly from standard congestion game theory, and (2) a number of key assumptions are shown to be easily relaxable as discussed above, therefore generalizing the orginal problem.

\section{B. Example Two}

In [9] the following problem was studied. A number of stationary mesh routers (802.11 nodes) share a fixed number of (potentially overlapping) channels in such a way that each router tries to minimize its sum interference. More specifically, there is an interference cost function $f\left(c_{1}, c_{2}\right)$ associated with a pair of channels $c_{1}$ and $c_{2}$, where $f\left(c_{1}, c_{2}\right)=$ $f\left(c_{2}, c_{1}\right)$. A router $i$ that selects channel $c_{1}$ pays such a cost if another router $j$ who is in $i$ 's interference set $\left(j \in N_{i}\right)$ selects channel $c_{2}$. Router $i$ updates its strategy by minimizing over all possible channel selections $c_{i}$ the sum $\sum_{j \in N_{i}} f\left(c_{i}, c_{j}\right)$ where $c_{j}$ is router $j$ 's selection at the time of update. It was shown in [9] that this greedy procedure reaches a stable state within a finite number of updates.

Below we show how this problem may be converted into an equivalent congestion game.

As before we have a set of players $\mathcal{N}$ and channels $\mathcal{C}$. Consider the virtual resource given by the tuple $\left(i, j, c_{i}, c_{j}\right)$, where $i$ and $j(i \neq j)$ are an ordered pair of users and $c_{i}$ and $c_{j}$ are two channels belonging to the set $\mathcal{C}$. A user $i$ has $C$ admissible strategies, each given by a set of resources:

$\sigma_{i} \in \Sigma_{i}=\left\{\left\{\left(i, j, c, c_{j}\right),\left(j, i, c_{j}, c\right): \forall j \in N_{i}, c_{j} \in \mathcal{C}\right\}: c \in \mathcal{C}\right\}$.

That is, when selecting channel $c$, a user $i$ uses all the resource tuples in which it appears as one of the two users with $c$ being its associated channel. There can be at most 2 users of a virtual resource, and its payoff (cost) is given by

$$
g_{\left(i, j, c_{i}, c_{j}\right)}(k)=\left\{\begin{array}{ll}
\frac{1}{2} f\left(c_{i}, c_{j}\right) & k=2 \\
0 & \text { otherwise }
\end{array} .\right.
$$

This specifies a congestion game, in which a user $i$ tries to minimize its total cost (interference) given by

$$
\begin{aligned}
g^{i}(\sigma) & =\sum_{j \neq i, j \in N_{i}}\left(g_{\left(i, j, c\left(\sigma_{i}\right), c\left(\sigma_{j}\right)\right)}(2)+g_{\left(j, i, c\left(\sigma_{j}\right), c\left(\sigma_{i}\right)\right)}(2)\right) \\
& =\sum_{j \neq i, j \in N_{i}} f\left(c\left(\sigma_{i}\right), c\left(\sigma_{j}\right)\right)
\end{aligned}
$$

over all possible choices of $\sigma_{i}$. This is exactly the same user objective as given in [9]. The potential function is given by

$$
\begin{aligned}
\phi(\sigma)= & \sum_{\left(i, j, c_{i}, c_{j}\right) \in \mathcal{R}} \sum_{k=1}^{n_{\left(i, j, c_{i}, c_{j}\right)}(\sigma)} g_{\left(i, j, c_{i}, c_{j}\right)}(k) \\
= & \frac{1}{2} \sum_{(i, j): j \neq i, j \in N_{i}}\left(g_{\left(i, j, c\left(\sigma_{i}\right), c\left(\sigma_{j}\right)\right)}(2)\right. \\
& \left.+g_{\left(j, i, c\left(\sigma_{j}\right), c\left(\sigma_{i}\right)\right)}(2)\right) \\
= & \frac{1}{2} \sum_{i=1}^{N} \sum_{j \neq i, j \in N_{i}} f\left(c\left(\sigma_{i}\right), c\left(\sigma_{j}\right)\right)
\end{aligned}
$$

a constant factor of the total interference in the system. The same convergence and local optimality results follow as in the previous example. The same discussion on generalization also applies here and is thus not repeated.

\section{Forward EngineEring: Client-Driven Distributed Spectrum Sharing Protocols}

We showed in the previous section how to reformulate two existing distributed spectrum sharing protocols as congestion games, by introducing virtual resources to model pairwise interference. This exercise indicates that we can obtain different spectrum sharing protocols by defining resources differently. In this section we examine a scenario of channel adaptation for base stations/access points and provide a new formulation based on a new resource definition. We will then discuss how to construct spectrum sharing protocols based on this.

As illustrated in Figure 2, consider multiple base stations with partially overlapping coverage areas. Each base station has a set of clients associated with it and it needs to select a good channel to operate in. We assume that a base station and its associated clients always operate on the same channel.

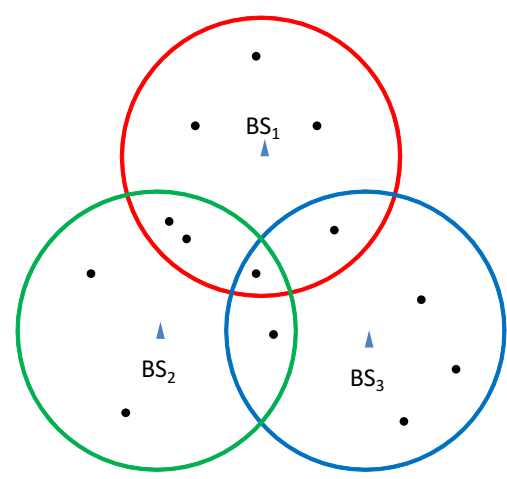

Fig. 2. Channel selection at base-stations. The dots indicate the clients. The circles indicate the coverage ranges of the base stations. 
Recall the spectrum-space block mentioned in Section II-B as an example defintion of a resource. We see that in this case the spectrum-space block is a natural and intuitive choice. By using a channel, a base station consumes a number of spectrum-space blocks, with the space dimension characterizing the coverage of the base station and the spectrum dimension characterizing the channel used by the base station. Then the spectrum-space blocks associated with the overlapping areas may be subject to congestion if multiple overlapping base stations choose the same frequency band. Below we define this congestion game more precisely.

We first note that within a base station's coverage area, congestion or interference is only perceived when a client exists at a particular location. In other words, it is not necessary to consider the entire space covered by a base station, but only where clients are located. Consequently, the spectrum-space block reduces to the following definition of resources: the set of all (client, channel) pairs. The resulting congestion game consists of the following.

- The players are the set of base stations $\mathcal{N}=$ $\{1,2, \ldots, N\}$.

- There are a set of clients $\mathcal{U}$. Each base station $i$ has a coverage area; the set of clients in $i$ 's coverage is denoted by $\mathcal{U}_{i}$. Conversely, for a client $u$, let $\mathcal{B}_{u}$ denote the set of base stations whose coverage ranges include $u$. Two base stations $i$ and $j$ are said to be neighbors if there is a client in both $i$ 's coverage range and $j$ 's coverage range.

- There are a set of channels $\mathcal{C}=\{1, \ldots, C\}$.

- The set of resources is the Cartesian product of $\mathcal{U}$ and $\mathcal{C}, \mathcal{R}=\{(u, c): u \in \mathcal{U}, c \in \mathcal{C}\}$.

- A player/base station has $C$ strategies, each given by the set of resources it consumes:

$$
\sigma_{i} \in \Sigma_{i}=\left\{\left\{(u, c): u \in \mathcal{U}_{i}\right\}, c=1, \ldots, C\right\} .
$$

As before, we will use $c\left(\sigma_{i}\right)$ to denote the channel that $i$ uses under strategy $\sigma_{i}$. In essence, a base station $i$ can choose among $C$ channels. When it is using channel $c$, it consumes resources $(u, c)$ for each client $u$ within its coverage range.

- Using resource $(u, c)$ incurs a payoff (cost) of $g_{(u, c)}\left(n_{(u, c)}\right)$, where $n_{(u, c)}$ is the total number of base stations using resource $(u, c)$.

- Each base station's objective is to maximize (minimize) its individual payoff (cost). Under the strategy profile $\sigma=\left(\sigma_{1}, \ldots, \sigma_{N}\right)$, base station $i$ 's payoff is:

$$
g^{i}(\sigma)=\sum_{u \in \mathcal{U}_{i}} g_{\left(u, c\left(\sigma_{i}\right)\right)}\left(n_{\left(u, c\left(\sigma_{i}\right)\right)}(\sigma)\right) .
$$

As this is now a congestion game, we have a stable system, in which any updating sequence (of frequency adaptation by the base stations) will lead to a local optimum of the potential function. We also note that our particular definition of resource suggests a client-driven approach, in which the clients measure their respective RF conditions and provide feed back to the base stations, who in turn make channel selections to optimize the above local payoff based on client reports and possibly information collected from other base stations.

Note that the local payoff a base station receives for using channel $c$ (given in (14)) takes into account all clients within its coverage area, including possibly clients associated with other base stations. This local payoff function suggests that a player (base station) is also concerned with the harm it causes to other players (base stations). Such "socially-responsible" behavior may seem at odds with the notion of a selfish player in a non-cooperative game. This is a direct consequence of our definition of resource, or resource expansion, whereby a client becomes a part of the resource. We see that in order to model mutual interference and at the same time to attain stability, resource expansion necessitates this type of sociallyresponsible behavior on the players' part; this is also the reason why we can align individual interests with a social objective. More discussion on the implementation of this is provided toward the end of this section.

We also note that each physical client $u$ corresponds to $C$ resources, $\{(u, c): c=1, \ldots, C\}$, one for each channel. The payoff for resource $(u, c)$ appears in the local optimization objectives of all base stations whose coverage area include $u$. This may seem a bit odd since client $u$ can only physically be in one of the channels, and thus it may seem that we should only be concerned with one resource $\left(u, c_{u}\right)$ where $c_{u}$ is the channel that $u$ is on. If the channels of the clients are fixed a priori, then it is indeed sensible to just consider one spectrum-space resource $\left(u, c_{u}\right)$ for each client $u$. However, in our present setup this is not fixed. Instead, $u$ 's associated base station is adapting the channel decision for all its clients. Without knowing which channel $u$ will use, our congestion game formulation considers all possible spectrum-space resources $\{(u, c): c=1, \ldots, C\}$.

Next we turn to practical protocols that implement the above game. From (14) we see that in order to make a channel switch decision, a base station needs to know for every channel the number of other base stations using it that also cover one or more of its clients. We describe two possible methods to implement this. The first method assumes that a client can directly communicate with all base stations whose coverage range includes it. In this method, clients switch channels from time to time and report for each channel the number of base stations it hears on the channel to the base stations. That would directly provide the payoff term for each resource $(u, c)$.

The second method assumes that neighboring base stations can communicate with each other via an inter-BS communication protocol, which can be supported by either over-theair signaling or out-of-band signaling (e.g., through a wired infrastructure where the BSs are attached to). In this case, the clients can listen on the channels for base station IDs and possibly other parameters (e.g., signal strength). But each client reports only to its own base station the set of all base stations it sees. The base stations then communicate with each other via the inter-BS communication protocol, such that each base station $i$ learns:

- the set of clients $\mathcal{U}_{i}$ in $i$ 's coverage area 
- for each client $u \in \mathcal{U}_{i}$, the set of base stations $\mathcal{B}_{u}$ whose coverage includes $u$

- the current operating channels of its neighbors.

We see that under this implementation the base stations essentially cooperate to stalize the system and reach a local optimal point, even though the congestion game framework is a non-cooperative one. As noted earlier this is a consequence of both resource expansion and the need to obtain sufficient information to play the game. This implementation is reasonable if we assume that all base stations belong to the same system (or administrative domain) and therefore may be assumed to follow the same protocol (user behavior). In this sense the congestion game model serves as a distributed way of reaching an equilibrum in a global system objective, which may be a much simpler implementation than adopting a centralized manager with centralized information collection. However, if these base stations belong to different systems, then it's questionable whether they are willing to cooperate, and whether the information they provide each other may be assumed true. In this latter case the congestion game may not be the most appropriate model and one may need to resort to mechanisms like pricing to enforce truth revelation.

\section{EXTENSIONS}

In this section we outline several possible extensions to the main results.

\section{A. Channel bundling}

Even though throughout the paper we have assumed the model that each user/player is limited to one channel at a time, the congestion framework can easily accommodate the more general model of allowing access to multiple channels simultaneously, or channel bundling. Indeed, in a congestion game, each user's strategy space is modeled as a set of strategies, where each strategy is a set of resources. We can model a user using multiple channels by defining their strategy spaces accordingly. In particular, we can also allow the bundling to be different for different users, and/or to be within a contiguous block.

However, what this framework does not immediately capture is the advantages and disadvantages of this type of channel bundling, e.g., by using two neighboring channels together one may get a higher rate than simply adding two individual rates. How to take this into account remains an interesting problem.

\section{B. Fractional frequency reuse}

In Section IV, we assumed that each base station chooses one channel for all its clients. A possible extension is to consider the technology of fractional frequency reuse, a technique currently considered by cellular standards such as 802.16. The concept is that in OFDMA systems different clients may be assigned different sets of sub-channels. Clients near the cell edge can be assigned fewer sub-channels that are carefully chosen to avoid interference from neighboring cells; in contrast, clients close to the cell center can make use of more sub-channels because they are less susceptible to cochannel interference. Similar as in the previous subsection, the congestion game framework can easily incorporate the technique of fractional frequency reuse, by allowing a base station to use different sets of bands for communicating with different clients.

\section{Modeling clients as players}

In Section IV, we modeled the base stations as players. We have assumed that client association is done independent of the base station's choice of channel, and that both the base station and its associated clients operate on the same channel. The problem there was for the base station to select a good channel given a fixed set of clients. A more sophisticated and more interesting scenario is to allow the clients freely choose which base station to associate with, depending on the latter's channel selection and perceived interference. In this case, presumably we will need to model the clients as strategic players as well. This will be further studied in a future work.

\section{User specific payoffs}

We have mentioned earlier that by using user-specific payoff functions one can model asymmetric interference relationship. This type of congestion games do not in general admit an exact potential function, and known results are weaker than the standard, non-user-specific payoff congestion games [3]. It would be interesting to see whether in the context of channel access our interference congestion games have better properties that may lead to stronger conclusions.

\section{CONCLUSION}

In this paper we have used the congestion game framework to study a number of problems that arise in spectrum sharing, in particular, in reducing the interference in such systems. Through two examples, we demonstrated that by using the notion of resource expansion we are able to better understand and generalize existing work. Specifically, for the problems studied in [11] and [9], we showed that they are fundamentally equivalent to a congestion game. As a result, known features of its solution immediately apply. In addition, they can be generalized and certain assumptions may be relaxed in a relatively straightforward way. We then showed that using the same methodology we can solve a base station channel adaptation problem.

\section{REFERENCES}

[1] R. Rosenthal, "A class of games possessing pure-strategy Nash equilibria," International Journal of Came Theory, vol. 2, pp. 65-67, 1973.

[2] B. Vöcking and R. Aachen, "Congestion games: Optimization in competition," Proceedings of the 2nd Algorithms and Complexity in Durham Workshop, H. Broersma, S. Dantchev, M. Johnson, and S. Szeider, Eds. Kings College Publications, London, 2006.

[3] I. Milchtaich, "Congestion Games with Player-Specific Payoff Functions," Games and Economic Behavior, vol. 13, no. 1, pp. 111-124, 1996.

[4] D. Monderer and L. S. Shapley, "Potential Games," Games and Economic Behavior, vol. 14, no. 0044, pp. 124-143, 1996.

[5] A. Fabrikant, C. Papadimitriou, and K. Talwar, "The complexity of pure Nash equilibria," Proc. 36th Annual ACM Symposium on Theory of Computing (STOC), pp. 604-612, 2004. 
[6] R. Etkin, A. Parekh, and D. Tse, "Spectrum Sharing for Unlicensed Bands," IEEE Journal on Selected Areas in Communications, vol. 25, no. 3, April 2007.

[7] J. Huang, R. A. Berry, and M. L. Honig, "Distributed Interference Compensation for Wireless Networks," IEEE Journal on Selected Areas in Communications, vol. 24, no. 5, May 2006.

[8] R. Menon, A. B. MacKenzie, R. M. Buehrer, J. H. Reed, "A gametheoretic framework for interference avoidance in ad-hoc networks," working paper.

[9] B-J. Ko, V. Misra, J. Padhye, and D. Rubenstein, "Distributed channel assignment in multi-radio 802.11 mesh networks," IEEE Wireless Communications and Networking Conference (WCNC), 2007.

[10] S. Adlakha, R. Johari, and A. Goldsmith, "Competition in wireless systems via Bayesian interference games," submitted for publication, 2008.

[11] B. Babadi and V. Tarokh, "A distributed dynamic frequency allocation algorithm," submitted for publication, 2008.

[12] W. Yu, G. Ginis, and J. M. Cioffi, "Distributed Multiuser Power Control for Digital Subscriber Lines," IEEE Journal on Selected Areas in Communications, vol 20, no. 5, June 2002. 